\title{
Exciton in closed and opened quantum dot
}

\author{
M.V.Tkach, Ju.O.Seti \\ Fedkovych Chernivtsi National University, 2, Kotsyubinskoho St., Chernivtsi, 58012, Ukraine*
}

Received September 4, 2006

\begin{abstract}
The theory of exciton spectrum in spherically symmetric states for the three- shell closed spherical quantum dot is proposed. The evolution of the exciton spectrum while varying the outer well thickness from zero (stationary spectrum of single closed spherical quantum dot) to infinity (quasistationary spectrum of a single open spherical quantum dot) is investigated. The mechanism of damping (semiwidth) of quasistationary states due to the redistribution over the energy levels of probability of exciton location in the space of two inner shells of nanosystem is studied. It is shown that the three shell closed spherical quantum dot of a rather big thickness of the outer well quite sufficiently and exactly reflects the basic properties of the quasistationary exciton spectrum in a single open spherical quantum dot.
\end{abstract}

Key words: spherical quantum dot, quasistationary state, exciton energy spectrum

PACS: 71.15.Dx, 73.21.La

\section{Introduction}

The present-day experimental possibilities of growing the multilayer nanoheterosystems, specifically, the technique of ion substitution, permit the fabrication and study of both closed and open quantum dots [1-3]. An important feature of the open system compared to the closed one is the possibility to control the additional channel of energy relaxation of qusiparticles due to their transition from the quantum dot to the outer medium. This makes it possible to develop ultra fast response detectors of radiation in the desired spectral region by choosing the proper geometric and physical parameters of the open multilayer quantum dots.

The theory of the spectra and wave functions of electrons and holes in single and multilayer spherical quantum dots (SQDs) embedded into an external medium is developed for both closed [2$4]$ and open [5,6] nanoheterosystems. Regarding the theory of excitons or the theory of interaction of electrons, holes, and excitons with phonons in closed SQDs, it is quite well developed in the context of various models for the phonon subsystem (especially, the model of dielectric continuum) for closed systems $[7,8]$. The results of the theory are in satisfactory agreement with the experimental data $[2,3]$.

In solving the problems of interaction of quasiparticles with quantum fields (phonons), the mathematical approaches of quantum mechanics [9] and the method of secondary quantization together with the Green functions theory [10] perform well for a closed system, but do not find direct use for the open one (with quasistationary states, in which the quasiparticles have a finite lifetime).

There is no theory of electron-phonon and exciton-phonon interactions in the open SQDs, since the wave functions of these quasiparticles in the quasistationary states are not involved in the complete system of normalized functions [9]. This fact does not allow us to use the methods of the quantum field theory studying the spectra renormalized by the interaction.

Studying a single open SQD, the basic theoretical difficulties can be avoided by treating the corresponding three-shell closed SQD with a very large width of the outer spherical well. Herein it is shown that at the increase of the outer well thickness, the exciton states of closed SQD cease to be stationary and when the thickness tends to infinity, the spectrum gradually becomes a quasistationary one.

*E-mail: theorphys@chnu.cv.ua 


\section{Exciton Hamiltonian, spectrum and wave functions in closed and open SQD}

The exciton energy spectrum and wave functions in semiconductor three-shell closed SQD with two potential wells and one barrier are under study. Geometrical parameters of the system are: the radius of inner well $\left(r_{0}\right)$, barrier thickness $\left(\Delta_{1}\right)$, and thickness of outer shell-well $\left(\Delta_{2}\right)$. It is clear that at $\Delta_{2} \rightarrow 0$ the three-shell nanosystem transits into the single closed SQD and at $\Delta_{2} \rightarrow \infty$ - into the single open SQD . Within the effective mass approximation and rectangular potential model for the electron and hole in spherical coordinate system (with the beginning in the center of SQD), as it is usually performed for the systems with close magnitudes of lattice constants for the composing parts of nanosystem [2-4] and assuming the electron and hole $(\mathrm{p}=\mathrm{e}, \mathrm{h})$ effective masses, potential energies and their dielectric constants to be known

$$
m_{p}\left(r_{p}\right)=\left\{\begin{array}{l}
m_{0,2} \\
m_{1,3}
\end{array} \quad, \quad U_{p}\left(r_{p}\right)=\left\{\begin{array}{l}
0, \\
U_{1,3}
\end{array}, \quad \varepsilon(r)= \begin{cases}\varepsilon_{0}, & r_{p}<r_{0}, r_{1}<r_{p}<r_{2} \\
\varepsilon_{1}, & r_{0} \leqslant r_{p} \leqslant r_{1}\end{cases}\right.\right.
$$

the Hamiltonian of exciton in three-shell closed SQD is obtained as

$$
H^{(\mathrm{c})}=H_{\mathrm{e}}^{(\mathrm{c})}\left(\vec{r}_{\mathrm{e}}\right)+H_{\mathrm{h}}^{(\mathrm{c})}\left(\vec{r}_{\mathrm{h}}\right)+V\left(\vec{r}_{\mathrm{e}}, \vec{r}_{\mathrm{h}}\right) .
$$

Herein, index (c) means that the system is closed and for the open system we shall further use index (o). The Hamiltonians of uncoupling electron $(p=e)$ and hole $(p=h)$ have the form

$$
H_{p}^{(\mathrm{c})}\left(r_{p}\right)=-\frac{\hbar^{2}}{2} \vec{\nabla} \frac{1}{m_{p}^{(\mathrm{c})}\left(r_{p}\right)} \vec{\nabla}+U_{p}^{(\mathrm{c})}\left(\vec{r}_{p}\right)
$$

and the potential energy of interacting quasiparticles in the system with dielectric constant $\varepsilon(r)$ is

$$
V\left(\vec{r}_{\mathrm{e}}, \vec{r}_{\mathrm{h}}\right)=-\frac{e^{2}}{\varepsilon\left(\vec{r}_{\mathrm{e}}, \vec{r}\right)\left|\vec{r}_{\mathrm{e}}-\vec{r}_{\mathrm{h}}\right|} .
$$

The Schrodinger equation with the exciton Hamiltonian (2) is not solved exactly but for the uncoupling electron and hole, the equation

$$
H_{p}^{(\mathrm{c})}\left(\vec{r}_{p}\right) \Psi_{p}^{(\mathrm{c})}\left(\vec{r}_{p}\right)=E_{p}^{(\mathrm{c})}\left(\vec{r}_{p}\right) \Psi_{p}^{(\mathrm{c})}\left(\vec{r}_{p}\right)
$$

taking into account the spherical symmetry, has the exact solution obtained in [11].

$$
\Psi_{p}^{(\mathrm{c})}\left(\vec{r}_{p}\right) \equiv \Psi_{\ell m}^{(c) p}\left(\vec{r}_{p}\right)=R_{\ell}^{(c) p}\left(r_{p}\right) Y_{\ell m}\left(\theta_{p}, \varphi_{p}\right), \quad \ell=0,1,2 \ldots, \quad m=0, \pm 1, \pm 2 \ldots
$$

where $Y_{\ell m}\left(\theta_{p}, \varphi_{p}\right)$ is the spherical function and $R_{\ell}^{\mathrm{cp}}\left(r_{p}\right)$ are the radial functions expressed through the Bessel and Neumann functions.

The fitting conditions for the wave functions and currents of probability densities continuity at all nanosystem interfaces together with the normalizing condition

$$
\begin{array}{ll}
\left.R_{i \ell}^{\mathrm{cp}}\left(r_{p}\right)\right|_{r_{p}=r_{i}}=\left.R_{i+1 \ell}^{\mathrm{cp}}\left(r_{p}\right)\right|_{r_{p}=r_{i}},\left.\quad \frac{1}{m_{p i}} \frac{\mathrm{d} R_{i \ell}^{p}\left(r_{p}\right)}{\mathrm{d} r_{p}}\right|_{r_{p}=r_{i}}=\left.\frac{1}{m_{p i+1}} \frac{\mathrm{d} R_{i+1 \ell}^{p}\left(r_{p}\right)}{\mathrm{d} r_{p}}\right|_{r_{p}=r_{i}}, \\
\int_{0}^{\infty}\left|R_{\ell}^{\mathrm{cp}}\left(r_{p}\right)\right|^{2} r_{p}^{2} \mathrm{~d} r_{p}=1
\end{array}
$$

determine $([11])$ the electron $\left(E_{n \ell}^{\mathrm{ce}}\right)$ and hole $\left(E_{n \ell}^{\mathrm{ch}}\right)$ energy spectra in the bound states $\left(U<U_{p}\right)$.

Using the information on the spectra and wave functions of uncoupling electron and hole, one can obtain the low energy part of exciton spectrum $\left(E_{n^{\prime}}^{n}\right)$ in spherically symmetric states 
$\left(\ell=\ell^{\prime}=0\right)$ within the framework of perturbation theory. According to this method, the exciton wave functions are written as

$$
\Psi_{n^{\prime}}^{n}\left(\vec{r}_{\mathrm{e}}, \vec{r}_{\mathrm{h}}\right)=\Psi_{n}^{\mathrm{ce}}\left(\vec{r}_{\mathrm{e}}\right) \Psi_{n^{\prime}}^{\mathrm{ch}}\left(\vec{r}_{\mathrm{h}}\right)=(4 \pi)^{-1} R_{n}^{\mathrm{ce}}\left(r_{\mathrm{e}}\right) R_{n^{\prime}}^{\mathrm{ch}}\left(r_{\mathrm{h}}\right) .
$$

The stationary exciton energy spectrum

$$
E_{n^{\prime}}^{n}=E_{g 0}+E_{n}^{\mathrm{e}}+E_{n^{\prime}}^{\mathrm{h}}+\Delta E_{n^{\prime}}^{n}
$$

is defined by the magnitude of the forbidden gap energy $\left(E_{g 0}\right)$ of the well, electron $\left(E_{n}^{\mathrm{e}}\right)$ and hole $\left(E_{n^{\prime}}^{\mathrm{h}}\right)$ energies in the corresponding states and interaction energy

$$
\Delta E_{n^{\prime}}^{n}=-\iint \frac{e^{2} \mathrm{~d}^{3} \vec{r}_{\mathrm{e}} \mathrm{d}^{3} \vec{r}_{\mathrm{h}}}{\varepsilon\left(\vec{r}_{\mathrm{e}}, \vec{r}_{\mathrm{h}}\right)\left|\vec{r}_{\mathrm{e}}-\vec{r}_{\mathrm{h}}\right|}\left|\Psi_{n}\left(\vec{r}_{\mathrm{e}}\right)\right|^{2}\left|\Psi_{n^{\prime}}\left(\vec{r}_{\mathrm{h}}\right)\right|^{2} .
$$

The exciton in an open nanosystem has the Hamiltonian (3), but here the outer medium turns out to be the well and there is no barrier at all. Thus, in the Hamiltonian of the open nanosystem, the effective masses and potential energies are as follows:

$$
m_{p}^{(\mathrm{o})}\left(r_{p}\right)=\left\{\begin{array}{l}
m_{p 0,2} \\
m_{p 1}
\end{array}, \quad U_{p}^{(\mathrm{o})}\left(r_{p}\right)=\left\{\begin{array}{ll}
U_{p 0,2}=0 & r_{p}<r_{0}, r_{1}<r_{p}<r_{2} \\
U_{p 1} & r_{0} \leqslant r_{p} \leqslant \infty
\end{array} .\right.\right.
$$

Now, the quasistationary exciton spectrum and its wave functions can be established within the S-matrix method using the general perturbation theory [9]. In this approach, the wave functions of spherically symmetric quasistationary states of uncoupling electron and hole are written as

$$
\Psi_{p}^{(0)}(\vec{r})=(4 \pi)^{-1 / 2} R_{p}^{(\circ)}\left(K_{p} r_{p}\right) .
$$

Their analytical expressions are presented in [11].

The exciton quasistationary spectrum and wave functions for the open SQD are obtained within the framework of the perturbation theory method [9]. Herein, it is assumed that to a certain magnitude of the exciton energy $\left(E_{\text {ex }}\right)$ of continuum spectrum, there corresponds an infinite number of electron and hole energies satisfying the condition

$$
E_{\mathrm{ex}}=\left(E_{\mathrm{e}} \pm E^{\prime}\right)+\left(E_{\mathrm{h}} \mp E^{\prime}\right), \quad 0 \leqslant \mathrm{E}^{\prime} \leqslant \min \left(E_{\mathrm{e}}, E_{\mathrm{h}}\right) .
$$

Thus, the radial part of exciton wave function $R_{\mathrm{ex}}^{(\mathrm{o})}$ is infinitely degenerated over the energy and can be determined by superposition

$$
R_{\mathrm{ex}}^{(\mathrm{o})}\left(E, r_{\mathrm{e}}, r_{\mathrm{h}}\right)=\int_{0}^{1} R_{\mathrm{e}}^{(\mathrm{o})}\left(E x, r_{\mathrm{e}}\right) R_{\mathrm{h}}^{(\mathrm{o})}\left(E(1-x), r_{\mathrm{h}}\right) \mathrm{d} x .
$$

The exciton wave functions in resonance spherically symmetric quasistationary states with the energy $E_{N^{\prime}}^{(\mathrm{o}) N}=E_{N}^{\mathrm{e}}+E_{N^{\prime}}^{\mathrm{h}}$ are as follows:

$$
\begin{aligned}
\Psi_{N^{\prime}}^{N}\left(E_{N^{\prime}}^{(\mathrm{o}) N}, \vec{r}_{\mathrm{e}}, \vec{r}_{\mathrm{h}}\right) & =(4 \pi)^{-1} R_{\mathrm{ex}}^{(\mathrm{o})}\left(E_{N^{\prime}}^{(\mathrm{o}) N}, r_{\mathrm{e}}, r_{\mathrm{h}}\right)= \\
& =(4 \pi)^{-1} \int_{0}^{1} R_{N}^{(\mathrm{o}) \mathrm{e}}\left(E_{N^{\prime}}^{(\mathrm{o}) N} x, r_{\mathrm{e}}\right) R_{N^{\prime}}^{(\mathrm{o}) h}\left(E_{N^{\prime}}^{(\mathrm{o}) N}(1-x), r_{\mathrm{h}}\right) .
\end{aligned}
$$

Finally, according to the general theory [9], the resonance quasistationary exciton energies $\left(E_{N^{\prime}}^{(o) N}\right)$ and semiwidths $\left(\Gamma_{N^{\prime}}^{N}\right)$ of the respective bands in the open SQD are fixed by the expressions

$$
E_{N^{\prime}}^{(\mathrm{o}) N}=E_{g 0}+E_{N}^{(\mathrm{o}) \mathrm{e}}+E_{N^{\prime}}^{(\mathrm{o}) \mathrm{e}}+\Delta E_{N^{\prime}}^{(\mathrm{o}) N}, \quad \Gamma_{N^{\prime}}^{(\mathrm{o}) N}=\Gamma_{N}^{(\mathrm{o}) \mathrm{e}}+\Gamma_{N^{\prime}}^{(\mathrm{o}) \mathrm{e}}+\gamma_{N^{\prime}}^{(\mathrm{o}) N},
$$


where

$$
E_{N}^{(o) p}=\operatorname{Repol} S^{p}\left(k_{N p}\right), \quad \Gamma_{N}^{(o) p}=-2 \operatorname{Impol} S^{p}\left(k_{N p}\right)
$$

are the resonance energies and semiwidths of quasistationary bands of uncoupling electron and hole, respectively $([11])$. The magnitudes $\Delta E_{N^{\prime}}^{(\mathrm{o})}$ and $\gamma_{N^{\prime}}^{(\mathrm{o})}$ are the corrections to the energy and semiwidths of exciton bands due to the interaction between electron and hole and are defined by

$$
\begin{aligned}
\Delta E_{N^{\prime}}^{(\mathrm{o}) N}+\mathrm{i} \gamma_{N^{\prime}}^{(\mathrm{o}) N}= & -\frac{e^{2}}{\varepsilon_{0} \sqrt{4 \pi}}(\operatorname{Re}-2 \mathrm{i} \operatorname{Im}) \int_{0}^{\infty} \int_{0}^{\infty} r_{\mathrm{e}}^{2} r_{\mathrm{h}}^{2} R_{\mathrm{ex}}^{2}\left(E_{N^{\prime}}^{(\mathrm{o}) N}, r_{e, h}\right)\left(\begin{array}{c}
r_{\mathrm{e}}^{-1}, r_{\mathrm{e}} \geqslant r_{\mathrm{h}} \\
r_{\mathrm{h}}^{-1}, r_{\mathrm{h}} \geqslant r_{\mathrm{e}}
\end{array}\right) \mathrm{d} r_{\mathrm{e}} \mathrm{d} r_{\mathrm{h}} \\
& \times\left(\int_{0}^{\infty} \int_{0}^{\infty} r_{\mathrm{e}}^{2} r_{\mathrm{h}}^{2} R_{\mathrm{ex}}^{2}\left(E_{N^{\prime}}^{(\mathrm{o}) N}, r_{e, h}\right) \mathrm{d} r_{\mathrm{e}} \mathrm{d} r_{\mathrm{h}}\right)^{-1}
\end{aligned}
$$

Thus, the quasistationary exciton spectrum in an open SQD is established.

\section{Evolution of exciton energy spectrum in spherically symmetric states from closed to open SQD}

The theory developed in the previous section allows us to study the exciton spectrum in spherically symmetric states $(\ell=0)$ depending on the thickness $\left(\Delta_{2}\right)$ of the external well of threeshell closed SQD. We can examine in detail the variation of exciton spectrum from stationary for the closed to the quasistationary for the open SQD. The numerical calculations were performed for the nanoheterosystem $\mathrm{HgS} / \mathrm{CdS} / \mathrm{HgS} / \mathrm{CdS}$ with the parameters: $m_{\mathrm{HgS}}^{\mathrm{e}}=0.036, m_{\mathrm{CdS}}^{\mathrm{e}}=0.2$, $U_{\mathrm{e}}=1350 \mathrm{meV}, m_{\mathrm{HgS}}^{\mathrm{h}}=0.044, m_{\mathrm{CdS}}^{\mathrm{h}}=0.7, U_{\mathrm{h}}=650 \mathrm{meV}$. These parameters satisfy the requirements of rectangular potential model due to the slight difference between the lattice constants of composition materials $\left(a_{\mathrm{HgS}}=5,851 \AA ; a_{\mathrm{CdS}}=5,818 \AA\right)$.

The evolution and properties of exciton spectrum is mainly caused by the spectra of uncoupling electron and hole. Therefore, figure 1 shows the results of calculation of the dependencies of stationary state energies $\left(E_{n 0}^{\mathrm{e}, \mathrm{h}(\mathrm{c})}\right)$ on the thickness $\left(\Delta_{2}\right)$ for small (a) and big (c) magnitudes and the probability of the location of these quasiparticles in the corresponding states

$$
W_{n 0}^{\mathrm{e}, \mathrm{h}(\mathrm{c})}=\int_{0}^{r_{0}+\Delta_{1}}\left|R_{n 0}^{\mathrm{e}, \mathrm{h}(\mathrm{c})}(r)\right|^{2} \mathrm{r}^{2} \mathrm{dr}
$$

for small (b) and big (d) thicknesses, respectively. Herein figure 1e presents the probabilities of electron and hole location $\left(W_{n 0}^{\mathrm{e}, \mathrm{h}(\mathrm{o})}\right)$ in the space of the open QD. In figure 1 one can see that the properties of electron and hole spectra are qualitatively equal and the quantitative difference is caused by the difference between their effective masses and potentials. The main features of the spectra are as follows. For the small thicknesses $\left(\Delta_{2}\right)$ of the order of $r_{0}+\Delta_{1}$ (figure 1) the stationary state energies $\left(E_{n 0}^{\mathrm{e}, \mathrm{h}(\mathrm{c})}\right)$ are decreasing with the increase of thickness. Herein, in the region of the energies corresponding to the energy levels of quasiparticle in the single SQD, one can see the anticrossings ("bottlenecks") arising due to the spherical symmetry of both inner and outer potential wells. Figure 1b presents the probabilities of the location of quasiparticles in stationary states of SQD at $\Delta_{2}=100 a_{\mathrm{CdS}}$. In the figure one can see that in the regions of anticrossings the probabilities are big $(\sim 1)$ while in the other regions of energies they are small $(\sim 0)$.

For the big magnitudes of thicknesses, when $\Delta_{2} \geqslant r_{0}+\Delta_{1}$ (figures 1c, d) the dependencies of $E_{n 0}^{\mathrm{e}, \mathrm{h}(\mathrm{c})}$ and $W_{n 0}^{\mathrm{e}, \mathrm{h}(\mathrm{c})}$ on $\Delta_{2}$ are very different from the previous case (figures $\left.1 \mathrm{a}, \mathrm{b}\right)$. Here, at the increase of $\Delta_{2}$, the energies $E_{n 0}^{\mathrm{e}, \mathrm{h}(\mathrm{c})}$ are also decreasing (figure 1c) but the "bottlenecks" are almost invisible. Nevertheless, the "memory" of the system about the location of anticrossings in the energy scale is already saved only in the probabilities $\left(W_{n 0}^{\mathrm{e}, \mathrm{h}(\mathrm{c})}\right)$ with the maxima just in the vicinity of the former anticrossings of quasistationary energy states of the open system (figure 1e). 


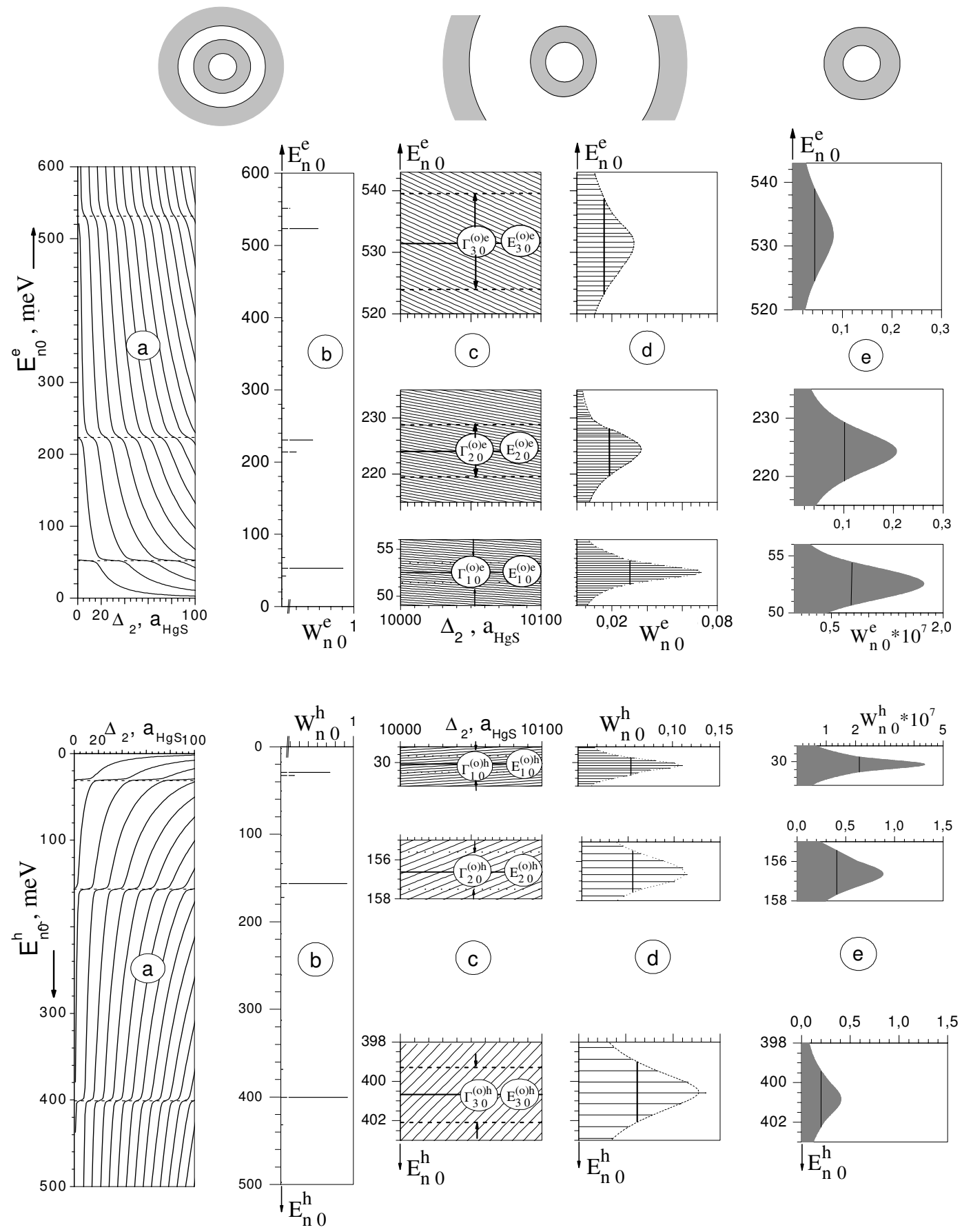

Figure 1. Dependencies of the energies $E_{n 0}^{\mathrm{e}, \mathrm{h}(\mathrm{c})}$ (a, c) on the thickness $\Delta_{2}$ and probabilities $W_{n 0}^{\mathrm{e}, \mathrm{h}(\mathrm{c})}(\mathrm{d})$ and $W_{n 0}^{\mathrm{e}, \mathrm{h}(\mathrm{o})}$ on the energies.

Herein, the distribution of probabilities $\left(W_{n 0}^{\mathrm{e}, \mathrm{h}(\mathrm{c})}\right)$ over the energies have the quasi-Lorentz character (figure 1d), similar to the analogous continuous distributions of probabilities $\left(W_{n 0}^{\mathrm{e}, \mathrm{h}(\mathrm{o})}\left(E^{\mathrm{e}, \mathrm{h}}\right)\right)$ in the open system (figure 1e).

Finally, in figures $1 \mathrm{c}, \mathrm{d}$, e one can see that at $\Delta_{2}=10000 a_{\mathrm{CdS}}$ among all the levels numerated 

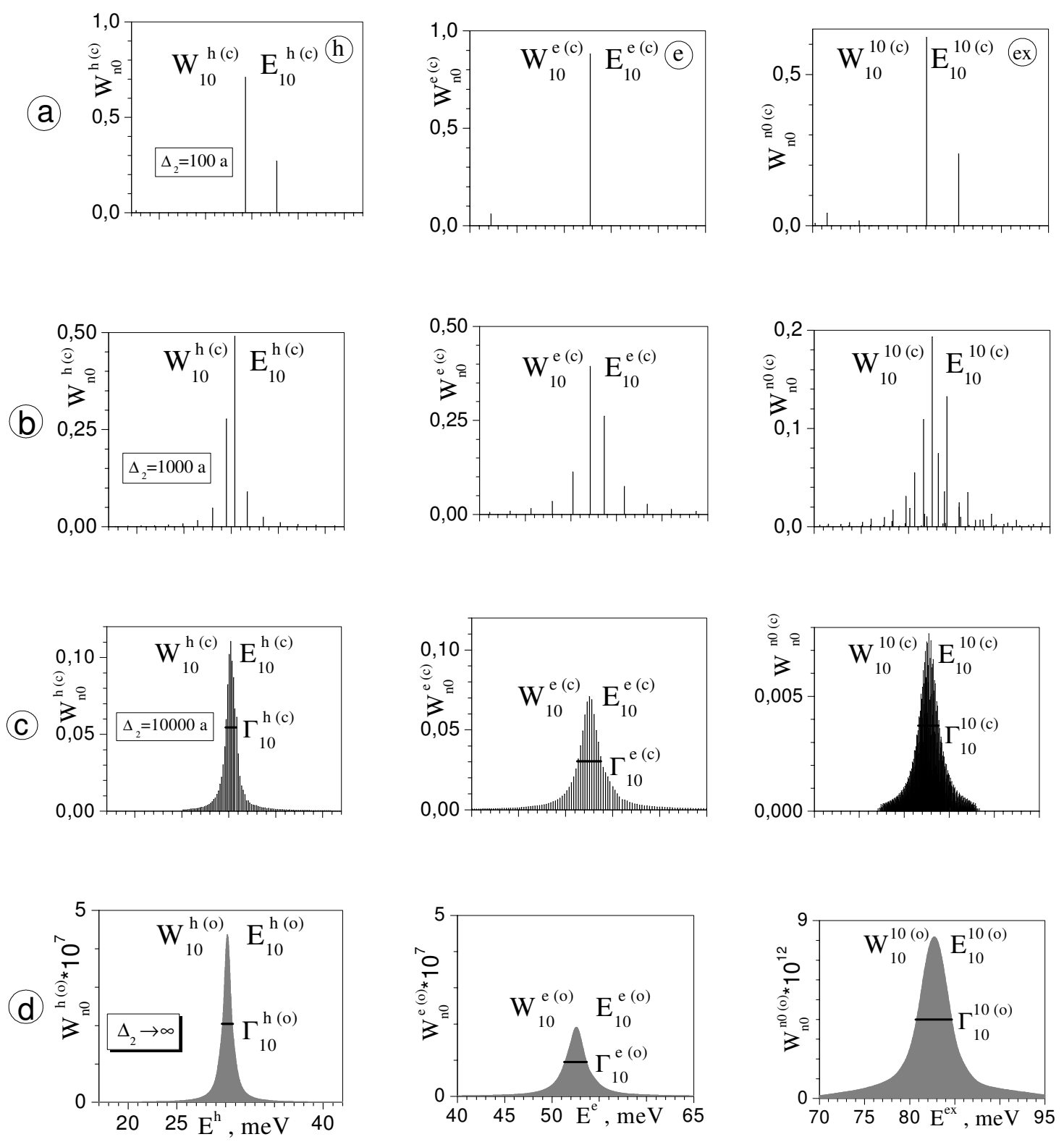

Figure 2. Dependencies of probabilities $W_{n}^{\mathrm{e}(\mathrm{c})}, W_{n}^{\mathrm{h}(\mathrm{c})}, W_{n}^{n(\mathrm{c})}$ and $W_{1}^{\mathrm{e}(\mathrm{o})}, W_{1}^{\mathrm{h}(\mathrm{o})}, W_{1}^{1(o)}$ on the energies for different thicknesses $\Delta_{2}$.

by $n$ quantum number, there are three levels fixed by the number $N=1,2,3$, corresponding to the stationary states with maximum probabilities $\left(W_{N}^{\mathrm{e}, \mathrm{h}(\mathrm{c})}\right)$. These energies $\left(E_{N}^{\mathrm{e}, \mathrm{h}(\mathrm{c})}\right)$ almost coincide with the resonance levels of the open $\operatorname{SQD}\left(E_{N}^{\mathrm{e}, \mathrm{h}(\mathrm{o})}\right)$. Due to the expression

$$
\lim _{\Delta_{2} \rightarrow \infty} E_{N}^{\mathrm{e}, \mathrm{h}(\mathrm{c})}=E_{N}^{\mathrm{e}, \mathrm{h}(\mathrm{o})}
$$

it is convenient to call these levels of closed system the "former resonance" ones. Figures 1d, $1 \mathrm{e}$ also proves that the widths of energy bands of closed SQD are almost equal to the widths of the quasistationary bands, i.e. $\lim _{\Delta_{2} \rightarrow \infty} \Gamma_{N}^{\mathrm{e}, \mathrm{h}(\mathrm{c})}=\Gamma_{N}^{\mathrm{e}, \mathrm{h}(\mathrm{o})}$.

The analysed behavior of the electron and hole spectra makes it possible to clarify the formation and evolution of exciton spectrum from the closed to the open SQD. The example of exciton 
spectrum and the formation of quasistationary bands at the variation of the thickness of SQD outer well $\left(\Delta_{2}\right)$ is presented in figures $2 \mathrm{a}-\mathrm{d}$. Figures $2 \mathrm{a}-\mathrm{c}$ show that at the increase of $\Delta_{2}$ (at $\left.r_{0}=20 \mathrm{a}_{\mathrm{HgS}}, \Delta_{1}=1 \mathrm{a}_{\mathrm{CdS}}\right)$, the distribution of probabilities of electron $\left(W_{n}^{\mathrm{e}}\right)$, hole $\left(W_{n}^{\mathrm{h}}\right)$ and exciton $\left(W_{n}^{n}\right)$ location in two inner shells of closed SQD in the vicinity of their lowest former resonance energies is $\left(\bar{E}_{1}^{\mathrm{e}(\mathrm{c})}, \bar{E}_{1}^{\mathrm{h}(\mathrm{c})}, \bar{E}_{1}^{1(\mathrm{c})}\right)$. Here (figure $2 \mathrm{~d}$ ) one can also see the distribution of probabilities $\left(W_{N}^{N}\right)$ of exciton location in the same inner shells in the states with the energies $E_{1}^{\mathrm{h}(\mathrm{o})}, E_{1}^{\mathrm{e}(\mathrm{o})}$ and $E_{1}^{1}=E_{1}^{\mathrm{h}(\mathrm{o})}+E_{1}^{\mathrm{e}(\mathrm{o})}$ from the vicinity of the lowest resonance state (without taking into account the energy of interaction $\Delta E_{1}^{1}$ and width of the forbidden gap $E_{g_{0}}$ ).
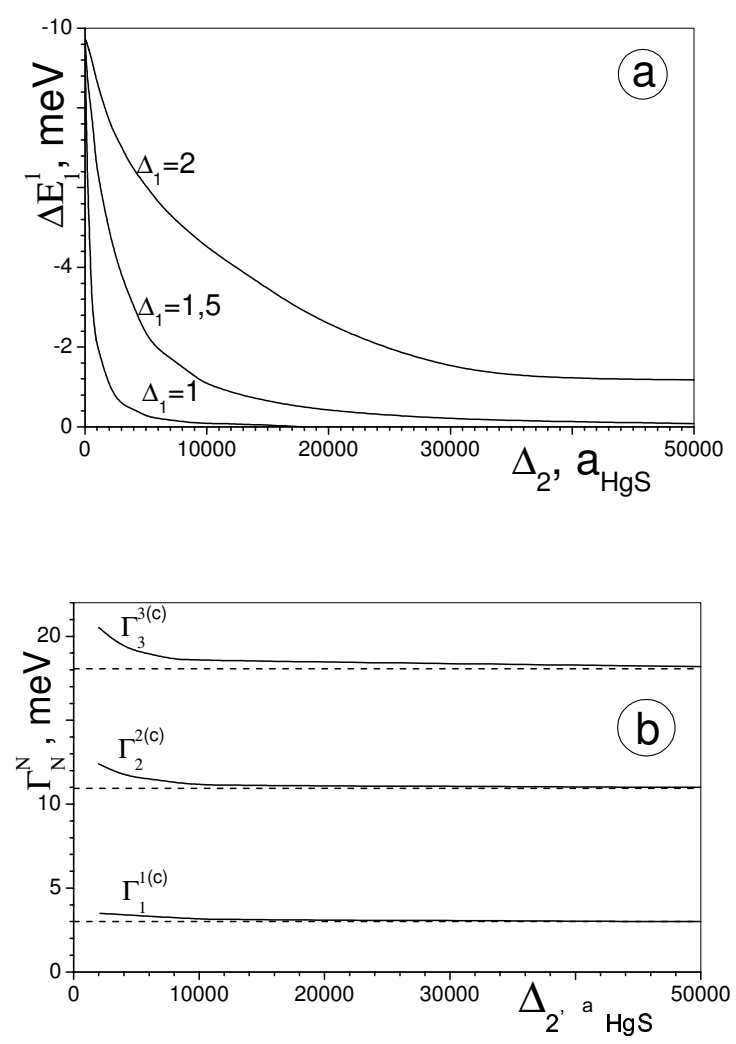

Figure 3. Dependence of binding energy $\left(\Delta E_{1}^{1}\right)$ at different thicknesses $\Delta_{2}$ (a) and semiwidth of discrete band $\left(\Gamma_{N}^{N}\right)$ at $\Delta_{1}=a_{\mathrm{HgS}}$ on the thickness $\Delta_{2}$.

Figure 2a proves that at the thickness $\Delta_{2}$ of the order of $r_{0}+\Delta_{1}\left(\Delta_{2}=100 \mathrm{a}_{\mathrm{CdS}}\right)$, the stationary spectra of all three quasiparticles are discrete with the big $(\sim 1)$ probabilities in the vicinity of resonance energies and small magnitudes of probability in a wider scale. At this thickness $\left(\Delta_{2}\right)$, for every quasiparticle (electron, hole, exciton), there does not exist the conception of discrete band semiwidth as an interval of energies where there are even two states around the resonance one satisfying the conditions

$$
2 W_{n \pm 1}^{\mathrm{e}, \mathrm{h}} \geqslant W_{n}^{\mathrm{e}, \mathrm{h}}=W_{N=1}^{\mathrm{e}, \mathrm{h}}, \quad 2 W_{n^{\prime} \pm 1}^{n \pm 1} \geqslant W_{n^{\prime}}^{n}=W_{N^{\prime}=1}^{N=1} .
$$

At $\Delta_{2}=1000 \mathrm{a}_{\mathrm{CdS}}$ the semiwidths of discrete bands do not arise yet, but their quasi-Lorentz contour becomes visible for all three quasiparticles. At $\Delta_{2}=1000 \mathrm{a}_{\mathrm{CdS}}$ (figure 2c) all discrete bands are characterized by semiwidths $\left(\Gamma_{1}^{\mathrm{h}(\mathrm{c})}, \Gamma_{1}^{\mathrm{e}(\mathrm{c})}, \Gamma_{1}^{1(\mathrm{c})}\right)$ and the former resonance energies $\left(\bar{E}_{1}^{\mathrm{h}}, \bar{E}_{1}^{\mathrm{e}}, \bar{E}_{1}^{1}\right)$. All these magnitudes for closed SQD almost do not differ from the resonance energies $\left(E_{1}^{\mathrm{h}}, E_{1}^{\mathrm{e}}, E_{1}^{1}\right)$ and semiwidths $\left(\Gamma_{1}^{\mathrm{h}}, \Gamma_{1}^{\mathrm{e}}, \Gamma_{1}^{1}\right)$ of the open SQD (figure $2 \mathrm{~d}$ ). Herein, $\Gamma_{1}^{1}=\Gamma_{1}^{\mathrm{h}(\mathrm{o})}+\Gamma_{1}^{\mathrm{e}(\mathrm{o})}$.

Figure 3 a presents the dependencies of electron and hole interaction energy $\left(\Delta \bar{E}_{1}^{1}\right)$ in the former resonance states $N_{\mathrm{e}}=N_{\mathrm{h}}=1$ on the thickness $\left(\Delta_{2}\right)$ at a different thickness of the barrier $\left(\Delta_{1}\right)$. In 
the figure one can see that at the increase of $\Delta_{2}$, the magnitude of $\Delta \bar{E}_{1}^{1}$ decreases. The calculation of the interaction energy $\Delta \bar{E}_{1}^{1}$ for the open SQD proves that at the varying of barrier thickness $\left(\Delta_{1}\right)$ in the range of several lattice constants, the magnitude of $\Delta \bar{E}_{1}^{1}$ is not bigger than $0.01 \mathrm{meV}$, since it is negligibly small.

Figure $3 \mathrm{~b}$ presents the dependence of semiwidth $\left(\bar{\Gamma}_{n}^{n}\right)$ of discrete exciton bands on the thickness of the external well $\left(\Delta_{2}\right)$ at $r_{0}=20 \mathrm{a}_{\mathrm{HgS}}, \Delta_{1}=1 \mathrm{a}_{\mathrm{CdS}}$. From this figure it is clear that the three discrete exciton bands start to be characterized by corresponding semiwidths when the thickness $\Delta_{2}$ becomes bigger than $10^{3} \mathrm{a}$ CdS. Herein, $\bar{\Gamma}_{N}^{N}$ for the closed system are always bigger than the respective semiwidths $\left(\Gamma_{N}^{N}=\Gamma_{N}^{\mathrm{h}}+\Gamma_{N}^{\mathrm{e}}\right)$ for the open SQD. At the increase of $\Delta_{2}$, the $\bar{\Gamma}_{N}^{N}$ values asymptotically tend to $\Gamma_{N}^{N}$, as it is supposed to be from physical considerations.

\section{Conclusions}

The theory of exciton spectrum in three-shell closed SQD has been developed for the first time. It permits to study the process of the formation and peculiarities of the evolution of spherically symmetric quasistationary states at the increase of the thickness of the outer well up to the sizes at which the SQD can be assumed as the open system.

It is established that for rather big thicknesses $\left(\Delta_{2} \gg r_{0}+\Delta_{1}\right)$ of the outer well of the closed three-shell SQD, in the vicinity of the resonance exciton energies $\left(E_{N^{\prime}}^{N}=E_{N^{\prime}}^{\mathrm{h}}+E_{N}^{\mathrm{e}}\right)$ of the single open SQD (close to the respective energies of the single closed SQD), the discrete exciton bands begin to form (with the former resonance energies $\left(\bar{E}_{N^{\prime}}^{N}\right)$ and semiwidths $\left(\bar{\Gamma}_{N^{\prime}}^{N}\right)$ ). At the increase of the thickness $\left(\Delta_{2}\right)$, the $\bar{E}_{N^{\prime}}^{N}$ and $\bar{\Gamma}_{N^{\prime}}^{N}$ magnitudes asymptotically tend to the resonance energies $E_{N^{\prime}}^{N}$ and semiwidths $\Gamma_{N^{\prime}}^{N}$ of exciton quasistationary states in a single open SQD.

Finally, it is worth noting that as far as the set of wave functions for the three-layer closed SQD is complete and orthonormalized, one can establish the theory of electron- and exciton-phonon interaction in the open SQD by choosing a rather big thickness of the outer well $\left(\Delta_{2}\right)$, ensuring the required exactness of the resonance energy $\left(E_{N^{\prime}}^{N}\right)$ and semiwidth $\left(\Gamma_{N^{\prime}}^{N}\right)$ of quasistationary spectrum of a single open SQD. This is the subject of our further investigation.

\section{Acknowledgements}

This work was financially supported by MPZ PP "Kolos".

\section{References}

1. Alferov Zh.I., Fiz. Tekh. Poluprovodn. (St. Petersburg), 1998, 32, 3[Semiconductors, 1998, 32, 1].

2. Schoss D., Mews A., Eychmuller A., Weller H. Phys. Rev. B, 1994, 49, 17078.

3. Mews A., Kadavanich A.V., Banin U., Alivisator A.P. Phys. Rev. B, 1996, 53, 13242.

4. Tkach M., Golovatskioe N., Mikhaleva M., Fartushinskioe R., Fiz. Tverd. Tela (St. Petersburg), 2001, 43, 1315 [Phys. Solid State, 2001, 43, 1370].

5. Tkach M., Golovatskioe N. Fiz. Tverd. Tela (St.Petersburg), 1999, 41, 2081 [Phys. Solid State, 1999, 41, 1911].

6. Tkach M., Holovatsky V., Voitsekhivska O. Physica E, 2001, 11, 17.

7. Klein M.C., Hache F., Ricard D., Flytzanis C. Phys. Rev. B, 1990, 42, 11143.

8. Tkach M., Holovatsky V., Voitsekhivska O., Mikhalyova M. Phys. Stat. Sol. (b), 1997, 207, 373; Studies, 2005, 9, 163 (in Ukrainian).

9. Baz A.I., Zeldovich Ya.B., Perelomov A.M. Scattering,Reactions and Decays in Nonrelativistic Quantum Mechanics, 2nd ed. Nauka, Moscow, 1971; IsraelProgram for Scientific Translations, Jerusalem, 1966.

10. Abrikosov A.A., Gorkov L.P., Dzyaloshinskioe I.E. Methods of Quantum Field Theory in Statistical Physics. Fizmatgiz, Moscow, 1962; Prentice-Hall, Englewood Cliffs, N.J., 1963.

11. Tkach M., Seti Ju. Fiz. Tekh. Poluprovodn (St. Petersburg), 2006, 40, 9 [Semiconductors, 2006, 40, 9]. 


\title{
Екситон у відкритій та закритій квантовій точці
}

\author{
М.В.Ткач, Ю.О.Сеті \\ Чернівецький національний університет ім. Юрія Федьковича, \\ вул. Коцюбинського 2, 58012, Чернівці
}

Отримано 4 вересня 2004 р.

\begin{abstract}
Вперше запропонована теорія екситонного спектра в сферично-симетричних станах тришарової закритої сферичної квантової точки (СКТ). Досліджено еволюцію спектра при зміні товщини зовнішнього шару-ями від нуля (стаціонарний спектр простої закритої СКТ) до безмежності (квазістаціонарний спектр простої відкритої СКТ). Досліджено механізм виникнення затухання (півширини) квазістаціонарних станів причиною якого є перерозподіл по енергетичних рівнях ймовірностей перебування екситона у межах двох внутрішніх шарів наносистеми. Показано, що тришарова закрита СКТ з достатньо великою товщиною зовнішньої ями з довільною необхідною точністю відтворює основні властивості квазістаціонарного екситонного спектра у простій відкритій СКТ.
\end{abstract}

Ключові слова: сферична квантова точка, квазістаціонарний стан, екситонний енергетичний спектр

PACS: 71.15.Dx, 73.21.La 
